\title{
Forced laminar-to-turbulent transition of pipe flows
}

\author{
By FRANZ DURST AND BÜLENT ÜNSAL \\ Institute of Fluid Mechanics, Friedrich-Alexander Universität Erlangen-Nürnberg, \\ Cauerstrasse 4, D-91058 Erlangen, Germany \\ buensal@1stm.uni-erlangen.de
}

(Received 25 October 2005 and in revised form 14 March 2006)

This paper presents the results of investigations into particular features of laminarto-turbulent transition of pipe flows. The first part considers transitional flows that occur 'naturally', i.e. without any forcing, when a critical Reynolds number is reached. Measurements are reported that were carried out to study the intermittent nature of pipe flows before they become fully turbulent. The second part of the paper concentrates on forced laminar-to-turbulent transition where the forcing was achieved by ring-type obstacles introduced into the flow close to the pipe inlet. The influence of the ring height was investigated and the results showed a dependence of the critical Reynolds number on the normalized height of the disturbances. The laminarto-turbulent transition was also investigated when caused by partially closing an iris diaphragm that permitted the flow to be forced to turbulence over short time intervals. Investigations of controlled intermittency became possible in this way and corresponding results are presented.

\section{Introduction, literature and aim of the work}

At least since the famous dye experiment of Reynolds (1883), there has been an overall understanding that fluids can flow through pipes in two distinct states, depending on the Reynolds number, Re, of the flow. Below a critical Reynolds number, the flow has the properties of a Hagen-Poiseuille flow and accidental disturbances that enter the flow are rapidly obliterated. The flow is referred to as being laminar and is said to be stable with respect to the introduced disturbances. As the Reynolds number is increased, the flow becomes increasingly sensitive to disturbances and it is observed that the fluid motion becomes irregular in time and space. This state of the flow is referred to as turbulent and its irregular motion at every location in the pipe is accompanied by an increase in friction factor, $c_{f}(R e)$. All this is well known and the overall properties of laminar and turbulent pipe flows can be considered as being available in the fluid mechanics literature.

In spite of the good knowledge of laminar and turbulent pipe flows, there are still a number of open questions regarding details of the two flow states. Most of these questions relate to the regime where the flow changes from the laminar to the turbulent state. Available studies suggest that the flow becomes turbulent at a critical Reynolds number in the range 2000-100 000 (e.g. see Schiller 1934; Ekman 1883; Pfenniger 1861), depending on the 'smoothness of the inlet' to the pipe flow. However, this general explanation is insufficient to explain the wide range of critical Reynolds numbers found in the literature for the occurrence of transitions. No clear 
picture exists of what 'smoothness of the inlet' means and how it relates to the critical Reynolds number at which transition occurs, i.e. there is no clear picture of what causes the flow to go from the laminar to the turbulent state in pipe flows.

One of the most detailed studies of the laminar-to-turbulent transition of pipe flows was carried out by Rotta (1956). He performed hot-wire velocity measurements through a pipe with length-to-diameter ratio $L / D=333$ and he was able to keep the air flow rate constant with a specially designed air supply valve, to prevent the flow rate oscillations usually induced by pressure drop changes, at the transitional Reynolds numbers. With three different inlet configurations, he obtained similar transitional Reynolds numbers. In Rotta's case, the flow was intermittent in the range $2000 \leqslant R e \leqslant$ 3000. Mean velocity profiles and intermittency were measured at different locations over the entire pipe length and, in this way, various details of the flow behaviour emerged.

Even more extensive work was carried out by Wygnanski \& Champagne (1973), Wygnanski, Sokolov \& Friedman (1975) and Rubin, Wygnanski \& Haritonidis (1980). They showed the existence of two different flow structures during the laminar-toturbulent transition of the flow, and referred to them as puffs and slugs, depending on their occurrence and dependence on the Reynolds number of the flow. Puffs were generated in their experiments by large disturbances at the inlet of the pipe and existed for the range $2000 \leqslant R e \leqslant 2700$ and slugs were generated by small disturbances for $R e \geqslant 3200$. These authors carried out detailed hot-wire velocity measurements in an $L / D=500$ pipe test rig, and it was claimed that a constant pressure drop for the air flow through the pipe was established for the investigations. The transitional Reynolds number measured in the investigations of Wygnanski and co-workers varied when the inlet nozzle-pipe configuration was changed or when mechanical disturbances were applied at the inlet. They measured mean velocities and turbulence intensities by using conditional sampling methods and ensemble averaging techniques to yield mean flow data. From these measurements, it was concluded that in their interior flow structure slugs had similar characteristics to the corresponding fully developed turbulent flow. The flow structures of the observed puffs differed from these typical characteristics of the fully developed state of the turbulent flow. Slugs also showed definite interfaces at their heads and tails. The length of a slug was of the same order as the pipe length and in some cases its duration corresponded to a time longer than the passage through the pipe length. Puffs, on the other hand, did not show a clear interface at their head, their mean velocities were roughly the same as the mean flow velocity of the pipe flow, the length of puffs stayed, on average, constant at a given Reynolds number and their duration was in general shorter than the passage time corresponding to the pipe length.

In investigations by Darbyshire \& Mullin (1995), a constant-mass-flow-rate test rig was used similar to that of Rotta (1956), but using water as the fluid medium. Disturbances were created by water injections with one and with six injector configurations at $L / D=70$ downstream from the pipe inlet. They observed similar flow structures as Wygnanski \& Champagne (1973). However, the main aim of their study was not the distinction of different flow structures but finding the threshold disturbance amplitude to initiate transition. They did not observe any turbulent motion below $R e \approx 1760$. From their investigations, Darbyshire \& Mullin (1995) concluded that the turbulent structures that they observed were independent of the kind of macroscopic disturbances that they applied to the flow.

In more recent studies by Draad, Kuiken \& Nieuwstadt (1998) and Hof, Juel \& Mullin (2003), investigated the amplitudes of the threshold disturbances yielding 


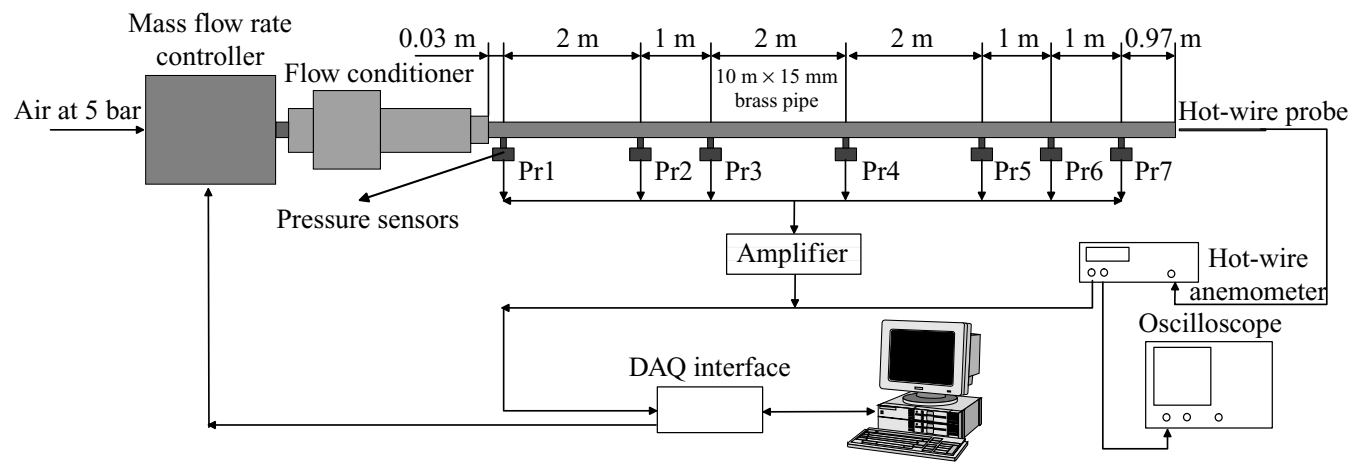

FIGURE 1. Schematic representation of the experimental test-rig.

laminar-to-turbulent transition of pipe flows and their dependence on the Reynolds number. In these studies, very similar to those of Darbyshire \& Mullin (1995), periodic blowing and sucking through holes in the wall were used to disturb the flow. The threshold amplitudes were defined based on the injected mean flow rate. Owing to differences in the experimental details, quantitative agreement with the results of Darbyshire \& Mullin (1995) was not obtained, as discussed by Trefethen et al. (2000).

The present investigations are based on the above-mentioned results of previous investigations and can be considered as an extension of the studies by Rotta (1956) and Wygnanski \& Champagne (1973). The investigations were preformed with a test rig including a $15 \mathrm{~mm}$ diameter brass pipe, with $L / D=667$. A mass flow control unit was applied to generate constant flow rates corresponding to predetermined Reynolds number. Along the pipe and over its entire length, seven pressure transducers were installed, which allowed one to observe the growth and decay of the transitional flow structures. At the exit of the pipe, hot-wire velocity measurements were conducted to record the state of the flow. In $\S 2$, the experimental test rig and the measurements conducted are described. Section 3 summarizes the measurements conducted under natural transition conditions. In $\S 4$, the results are given for ring-type obstacles at the pipe inlet. The results for the iris-diaphragm-triggered turbulence are summarized in $\S 5$, and in $\S 6$ conclusions and an outlook for future research in this field are presented.

\section{Test rig and measuring equipment}

To carry out investigations of various aspects of laminar-to-turbulent transitions of pipe flows, a test rig was set up with the major components shown in figure 1. Its main part consisted of a brass pipe of $15 \mathrm{~mm}$ diameter and total length $10 \mathrm{~m}$, corresponding to $L / D=666.7$. The flow of air was provided by a high-pressure supply line connected to a mass flow rate control unit of the type described by Durst et al. (2003). This unit is shown in figure 2, providing information about the theoretical background of the operation of the control valve and of its control electronics. This figure provides information on all the essential parts that control the mass flow rate supplied to the brass pipe test rig to within $\pm 1 \%$ of the required flow. The entire system operates in such a way that pressure changes in the transitional flow regime do not influence the mass flow through the pipe.

The flow rate control unit was driven by a pressure supply of 5 bar imposed in its pre-chamber. This generated a flow through a critical nozzle, providing a constant 
(a)
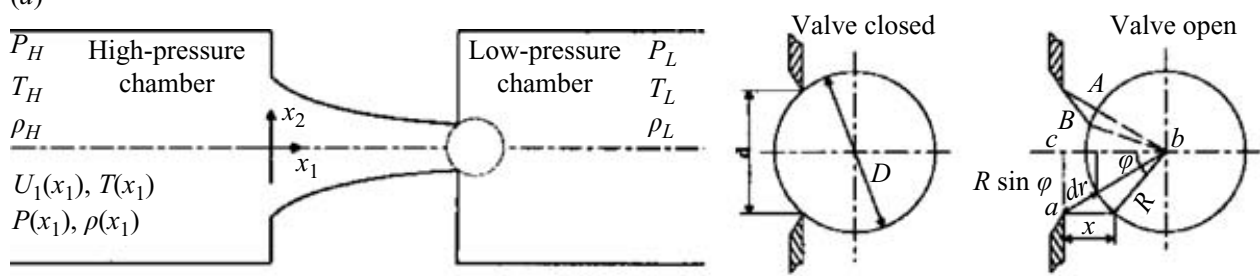

$$
\dot{m}=F\left(P_{H}, T_{H}, \kappa, R^{2}, x, \varphi\right) \quad \dot{m}=\frac{P_{H}}{\sqrt{\operatorname{Re} T_{H}}}\left[\sqrt{\frac{2 \kappa}{\kappa+1}}\left(\frac{2}{\kappa+1}\right) \frac{1}{\kappa-1}\right] \frac{\pi R^{2}}{\sqrt{1+2 \frac{x}{R} \cos \varphi}}\left[\left(\frac{x}{R}\right) \sin 2 \varphi\right]
$$

(b)
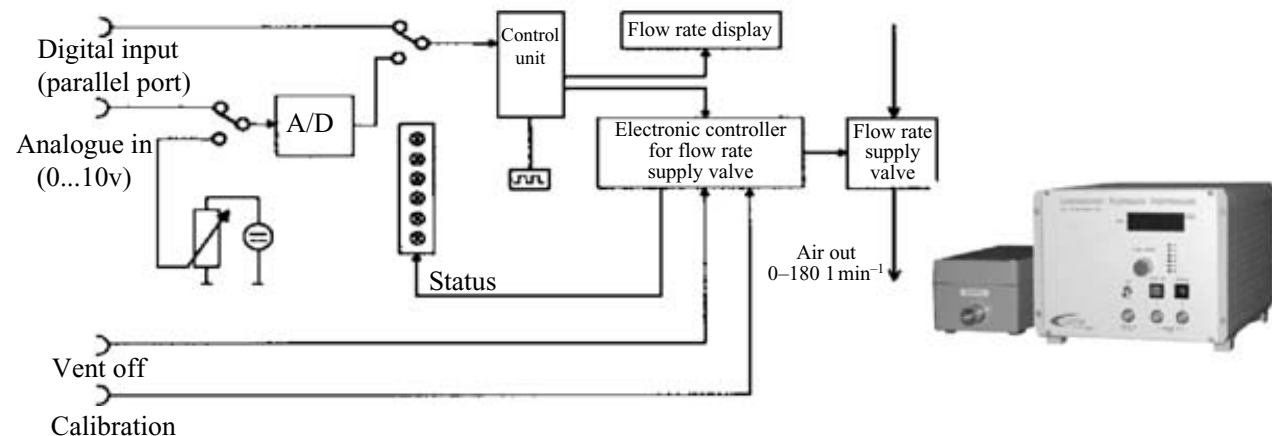

FIGURE 2. (a) Basic fluid flow considerations and relationship for $\dot{m}(x) ;(b)$ control circuit, actual mass flow rate controller.

(a)

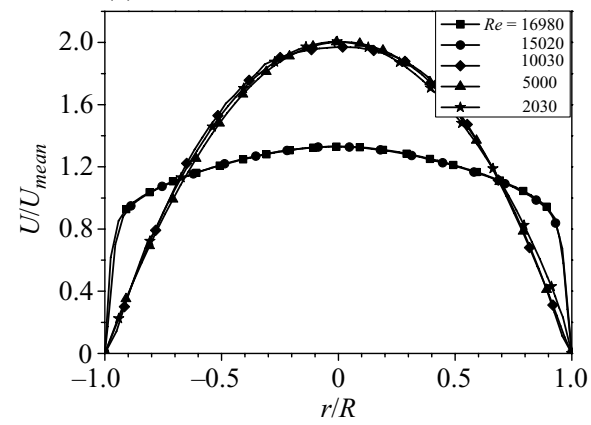

(b)

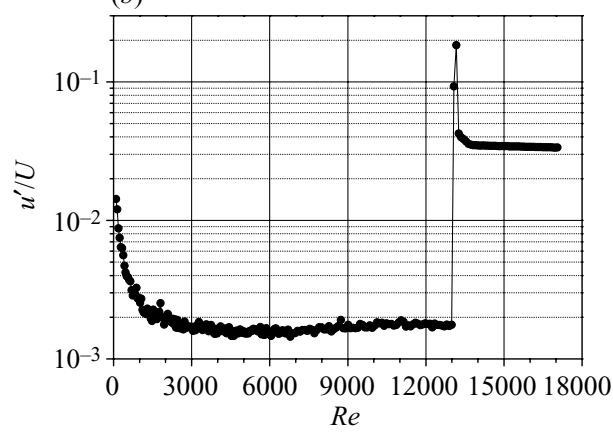

FIGURE 3. (a) Selected mean velocity profiles across the pipe and $(b)$ centreline turbulence intensity variation with Reynolds number at the pipe exit.

mass flow rate proportional to the nozzle area, as shown in figure 2. Pressure variations in the pre-chamber of the mass flow rate control unit were recorded and used by the electronics sketched in figure 2 to ensure that mass flow rate $\dot{m}=$ constant was achieved for each set flow rate. Hence the nozzle flow area was set to achieve a constant Reynolds number by a linear drive, as explained by Durst et al. (2003). In this way, the test rig, sketched in figure 1, permitted pipe flow investigations under steady flow conditions.

To provide a well-controlled inlet flow to the pipe, various flow conditioners were applied and the final one chosen was installed. As shown in figure 3, it permitted 
the laminar flow regime of the pipe flow to be monitored up to $R e \approx 13000$. By repeating experiments it was shown that the Reynolds number range at which the laminar-to-turbulent transition occurred was highly repeatable within $\pm 2 \%$ of the appropriate Reynolds number setting. Typical velocity profiles, measured at the exit of the pipe for different Reynolds number, are also given in figure 3. For the highest Reynolds number, still lying in the laminar regime, the turbulence level of the flow is indicated in figure 3 , showing a turbulence level of $u^{\prime} / U \approx 0.2 \%$. This turned out to be sufficiently small to carry out the investigations described in this paper.

To carry out instantaneous pressure measurements along the pipe, seven pressure transducers were installed, each with an operating range of $\pm 20 \mathrm{mbar}$ and $1 \mathrm{kHz}$ time resolution. The locations of the pressure transducers along the pipe are shown in figure 1 registering the occurrence of laminar or turbulent flow, at that particular location along the pipe, of course only with the local resolution given by these locations. This turned out to be sufficient for the laminar-to-turbulent flow transitional investigations described in this paper.

As already mentioned, a hot-wire anemometer set-up was employed at the exit of the pipe to carry out local velocity measurements. It included a traversing system and a single wire probe connected to a DISA 55 M01 constant-temperature hot-wire anemometer. To obtain velocity profile measurements, the vertical motion of the traversing system was activated and controlled through a PC. All pressure transducer and hot-wire anemometer output was connected to a 16-channel 16-bit $333 \mathrm{kHz}$ data acquisition card for simultaneous measurements of velocity and corresponding wall pressures. The output flow rate of the mass flow rate controller was also set by the PC and a special software program ensured that the entire measurements could be carried out in a well-controlled manner. Various sub-programs within the data acquisition system were written to carry out the processing of all data to yield the flow and pressure information provided in this paper.

The above-described properties of the flow control unit and the test-rig make it clear that this flow facility is ideally suited to studying transitional flows. When turbulent transition occurs in a pipe, the pressure drop changes and the unit, which the authors have built for this purpose, automatically adjusts immediately to take this increased pressure drop into account to yield a pipe flow with a constant mass flow rate. Wygnanski and co-workers had to achieve this by running their test-rig at high pressures so that the pressure changes due to laminar-to-turbulent transition did not have a big effect on their results.

\section{Studies of natural transition}

The test rig (figure 1) represented an ideal test section for studying the naturally occurring laminar-to-turbulent transition. As mentioned in the previous section, initial experiments showed that for the finally chosen inlet flow conditioner the laminar-toturbulent transition took place at a Reynolds number of around 13000. Below this critical value, the turbulence intensity, at the centreline of the flow, remained below $0.2 \%$, representing the background turbulence of the test facility. With increasing Reynolds numbers, the flow became intermittently turbulent, yielding instantaneous velocity records at the end of the pipe, as indicated in figure 4 . The instantaneous centreline velocity is shown in figure 4 for various Reynolds numbers, $R e \geqslant 12990$, indicating the onset of intermittency for $\mathrm{Re} \geqslant 13080$. The flow was fully turbulent, i.e. intermittency free, for $\mathrm{Re} \geqslant 13300$. The intermittency of the flow resulted in slug-like disturbances, as observed by Wygnanski \& Champagne (1973) and Wygnanski et al. 

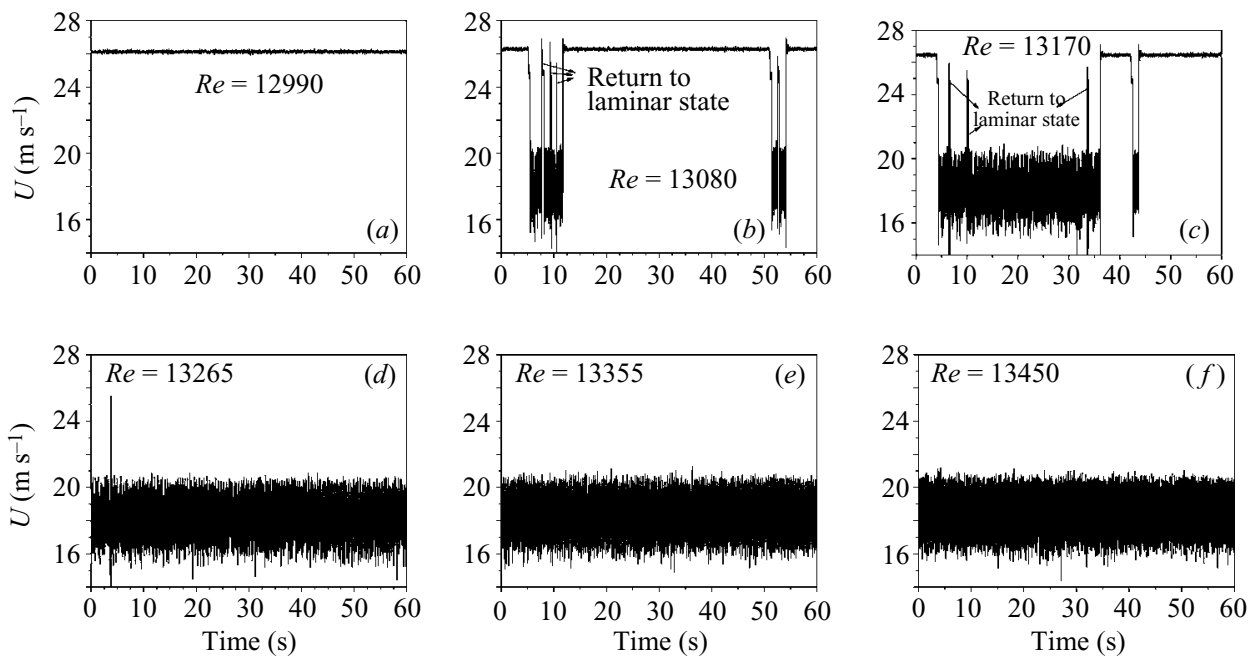

FIGURE 4. Instantaneous centreline velocity plots around the transitional Reynolds numbers for natural transition to turbulence.
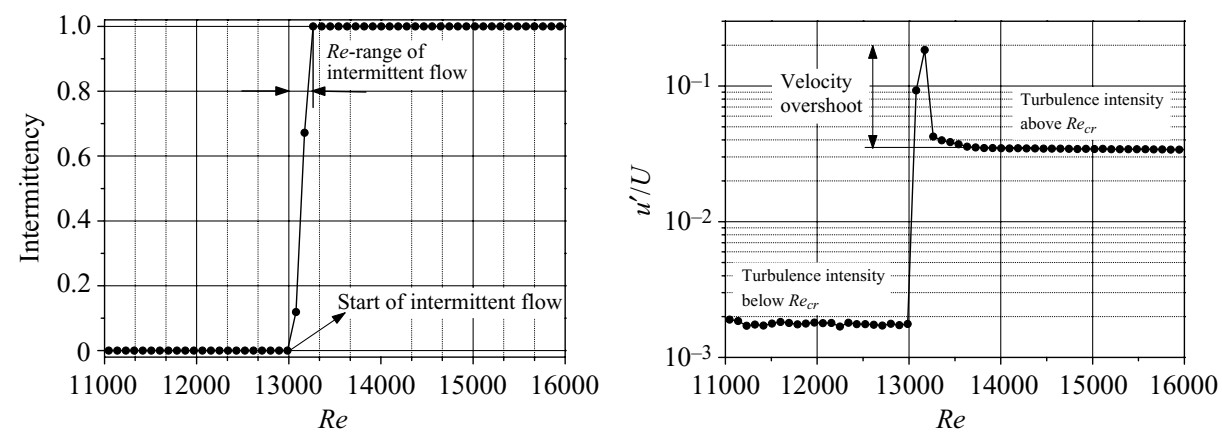

FIGURE 5. Intermittency at the pipe exit and corresponding turbulence intensity as a function of Reynolds number.

(1975), consisting of time-varying flow periods with 'jumps' from the laminar to the turbulent flow state and vice versa.

From the intermittency function (ratio of total turbulent state duration to overall measurement time) of figure 5 and the corresponding turbulent intensities, as a fuction of Reynolds number, one can see that high turbulent intensity values are measured owing to high velocity jumps between laminar and turbulent velocity profiles as indicated in figure $4(b)$ and $4(c)$. The turbulent intensity overshoots shown for the axial velocity fluctuation in figure 5 are due to this jump-like flow behaviour.

These velocity and intermittency measurements permit a good insight into the overall flow structures that occurred in the Reynolds number range of laminarto-turbulent transition, although all information was deduced from the velocity measurements at the outlet of the pipe. The corresponding pressure measurements in figure $6(a)$ show the pressure variation with time in the Reynolds number range when slugs were formed. Figure $6(b)$ shows the corresponding velocity signal at the 

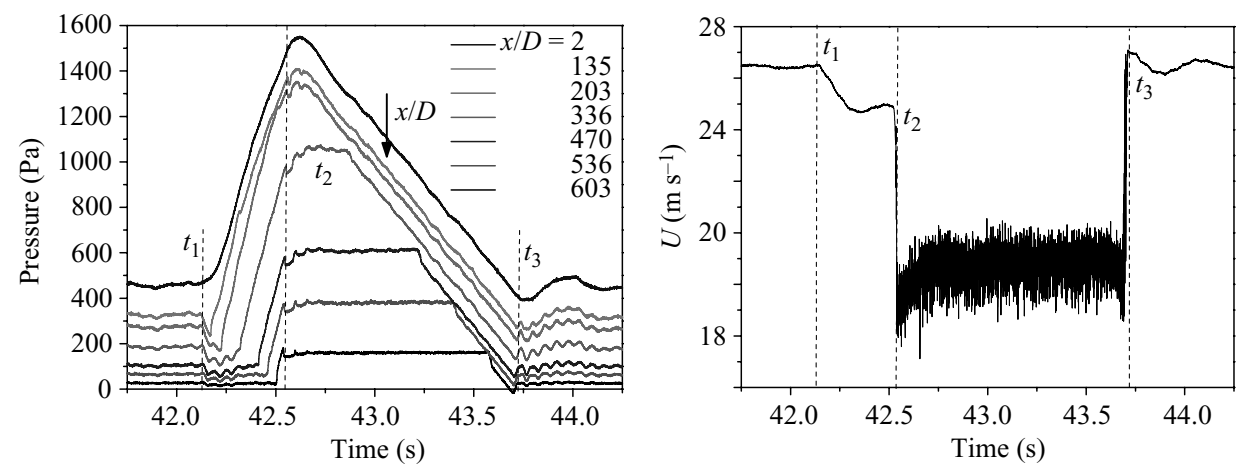

FiguRE 6. Measured pressure and velocity waveforms of a single slug structure. The pressure waveforms indicate the travel of a slug structure through the pipe length and the velocity waveform shows the slug structure at the pipe exit.

end of the pipe. Looking at both time traces permits the following information to be deduced:

(i) The slug flow starts to develop from the inlet of the pipe. It is swept downstream and fills the pipe as time proceeds. This development occurs within the time period $t_{1}$ to $t_{2}$.

(ii) At time instant $t_{2}$, the slug front reaches the pipe exit and at this time the slug length in the pipe is a maximum.

(iii) In the time period from $t_{2}$ to $t_{3}$, the slug tail moves through pipe and leaves the pipe at time instant $t_{3}$.

The combined velocity and pressure information is shown in figure 7. Figure 7(a) shows the r.m.s. values of longitudinal velocity fluctuations at the axis of the pipe as a function of Reynolds number. The corresponding local turbulence intensity is presented in figure $7(b)$, showing the increase at $\mathrm{Re} \approx 13000$, when the laminar-toturbulent transition occurs. The overshoot in the r.m.s. velocity fluctuations is clearly visible, caused by the laminar-to-turbulent and turbulent-to-laminar velocity jumps that occur at the beginning and end of the slug-like flows. Thereafter, i.e. for $\mathrm{Re} \approx$ 13500 , the region of fully developed turbulent pipe flow exists at all times with its well-known turbulent flow properties.

This flow behaviour is also reflected by the friction factor $f=2 \tau_{w} /\left(\rho \widetilde{U}^{2}\right)$ that corresponds for $R e \leqslant 13000$ to the theoretically predicted relationship $f_{\text {lam }}=64 / R e$, as indicated in figure $7(c)$. For $R e \geqslant 13500$, the experimentally obtained friction factor is described well by the relationship $f_{\text {turb }}=0.3614 / R e^{1 / 4}$. It is interesting that the peak in the r.m.s. value of the longitudinal velocity fluctuation on the axis of the pipe flow does not result in a corresponding overshoot of the friction factor.

Overall, the experimental results described in this section clearly reveal that the experimental test facility permitted laminar-to-turbulent pipe flows of air to be investigated in a well-controlled manner yielding highly repeatable results. These results showed that the investigated laminar-to-turbulent flow transition apparently occurred as a result of disturbances at the pipe inlet, i.e. transition always started at the pipe inlet. This phenomenon will require further experimental and theoretical studies. However, the results obtained so far are sufficient to permit the study of the forced laminar-to-turbulent flow transition described in the next two sections. They were used in the investigations to distinguish between puff-like and slug-like transition 

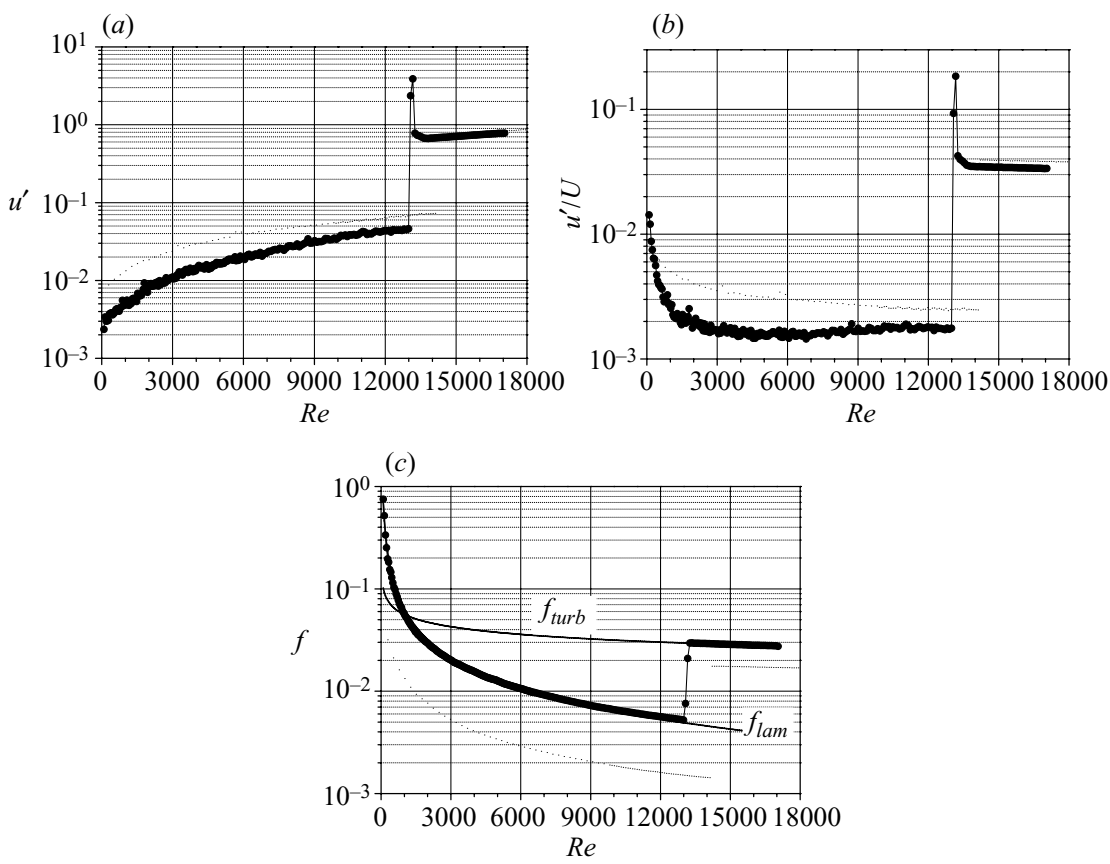

FIGURE 7. (a) RMS velocity, (b) turbulence intensity and (c) friction factor change with $R e$ for natural laminar-to-turbulent transition. Velocities were measured at the centreline of the pipe exit. Pressures were measured by the last two transducers.

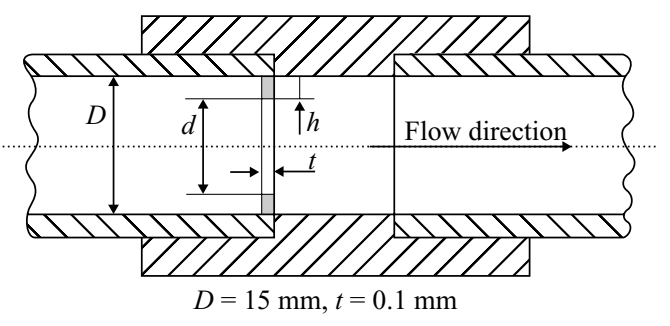

\begin{tabular}{ccc}
\hline \% Blockage & $d(\mathrm{~mm})$ & $h / t$ \\
7.5 & 14.427 & 2.87 \\
10 & 14.230 & 3.85 \\
15 & 13.829 & 5.85 \\
20 & 13.416 & 7.92 \\
25 & 12.990 & 10.05 \\
30 & 12.550 & 12.25 \\
40 & 11.619 & 16.91 \\
\hline
\end{tabular}

FIGURE 8. Sketch showing the application the ring-type obstacles of diameter $d$ which were placed at the exit of the flow conditioner and a table showing the dimensions of the rings with different blockage ratios.

to turbulence. The flow structures are so distinctly different that a differentiation between puffs and slugs represented no problem in all phases of the investigations.

\section{Transition forced by ring obstacles}

It is common practice in turbulent pipe flow research to utilize triggering devices upstream of the investigated flow to force the flow to become turbulent in a more defined manner. For turbulent pipe flow, ring-type obstacles of the kind shown in figure 8 are commonly employed. This encouraged the authors to look at such triggering devices and to investigate their influence on the laminar-to-turbulent flow transition in pipes. Various rings were used and were mounted between the flow control unit shown in figure 1 and the inlet of the pipe. To ensure high concentricity of the inner and outer diameters $d$ and $D$ of the rings, laser cutting and special machining were employed 


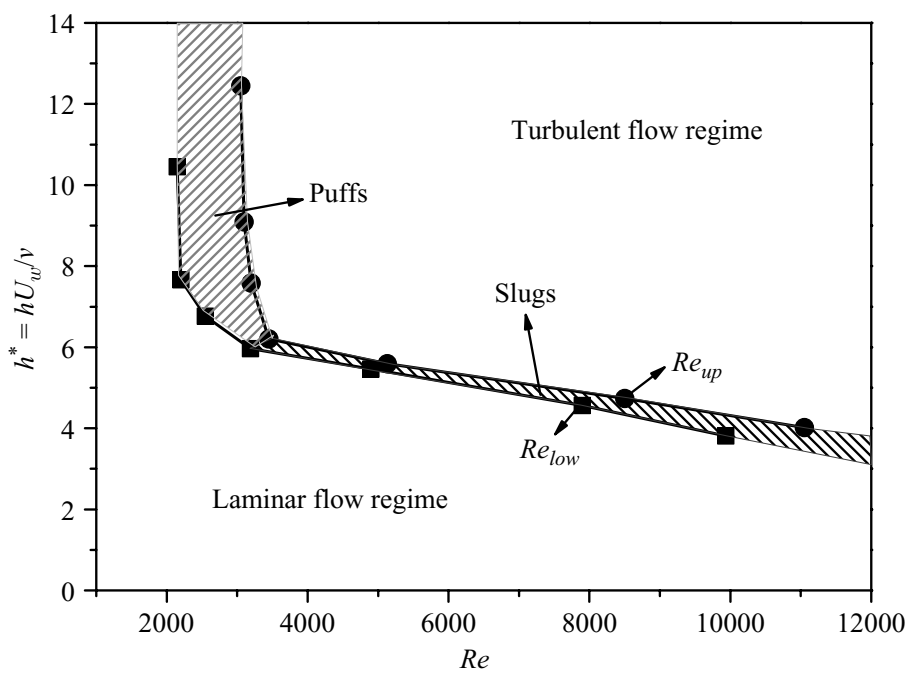

FIGURE 9. The variation of lower $\left(R e_{l o w}\right)$ and upper critical $\left(R e_{u p}\right)$ Reynolds number with dimensionless ring-height $h^{*}$. The dashed areas correspond to the transitional regime the with slugs and puffs were indicated separately with different shadings.

to ensure that both diameters to had common centre and also to be accurate within $\pm 10 \mu \mathrm{m}$. Different blockage ratios were employed, as indicated in figure 8 . For each blockage ratio, a separate set of investigations was carried out utilizing the velocity and pressure measurement facilities employed for the transition studies described in $\S 3$. Hence, the state of the flow in the pipe was deduced from pressure gradient measurements and turbulence intensity records at the end of the pipe.

The investigations carried out with different obstacles revealed that the critical Reynolds number for the laminar-to-turbulent flow transition decreased with increasing obstacle height. This is shown in figure 9, providing a summary of the transitional flow results.

For the flows triggered by obstacles at the pipe inlet, investigations were performed on the critical Reynolds number occurring for each obstacle. The results clearly showed, for both the pressure and velocity measurements, that the laminar-toturbulent transition occurred over a small finite Reynolds number range, indicated by $R e_{\text {low }}$ and $R e_{\text {up }}$. Figure 10 shows how extrapolated values of the turbulence intensity measurements to the laminar state of the flow, $R e_{\text {low }}$, and to the turbulent state of the flow, $R e_{u p}$, were employed to define the Reynolds number range within which flow transition occurs.

These investigations permit the critical ring obstacle height that is needed to turn the flow from the laminar to turbulent state to be defined. Dimensional analysis suggests that the normalized height $h^{*}$ can be introduced as

$$
h^{*}=\frac{h U_{\tau}}{v}
$$

to characterize the critical height, where $h$ is the obstacle height, $U_{\tau}=\sqrt{\tau_{w} / \rho}$ the wall friction velocity of the laminar flow, $v$ the kinematic viscosity, $U_{\text {mean }}$ the mean velocity of the pipe flow and $D$ the pipe diameter. Utilizing $h^{*}=f(R e)$ in figure 9 yields a clear separation between the laminar and turbulent flow regimes of the pipe flow when triggered by ring-type obstacles. The decrease in the normalized obstacle height with 

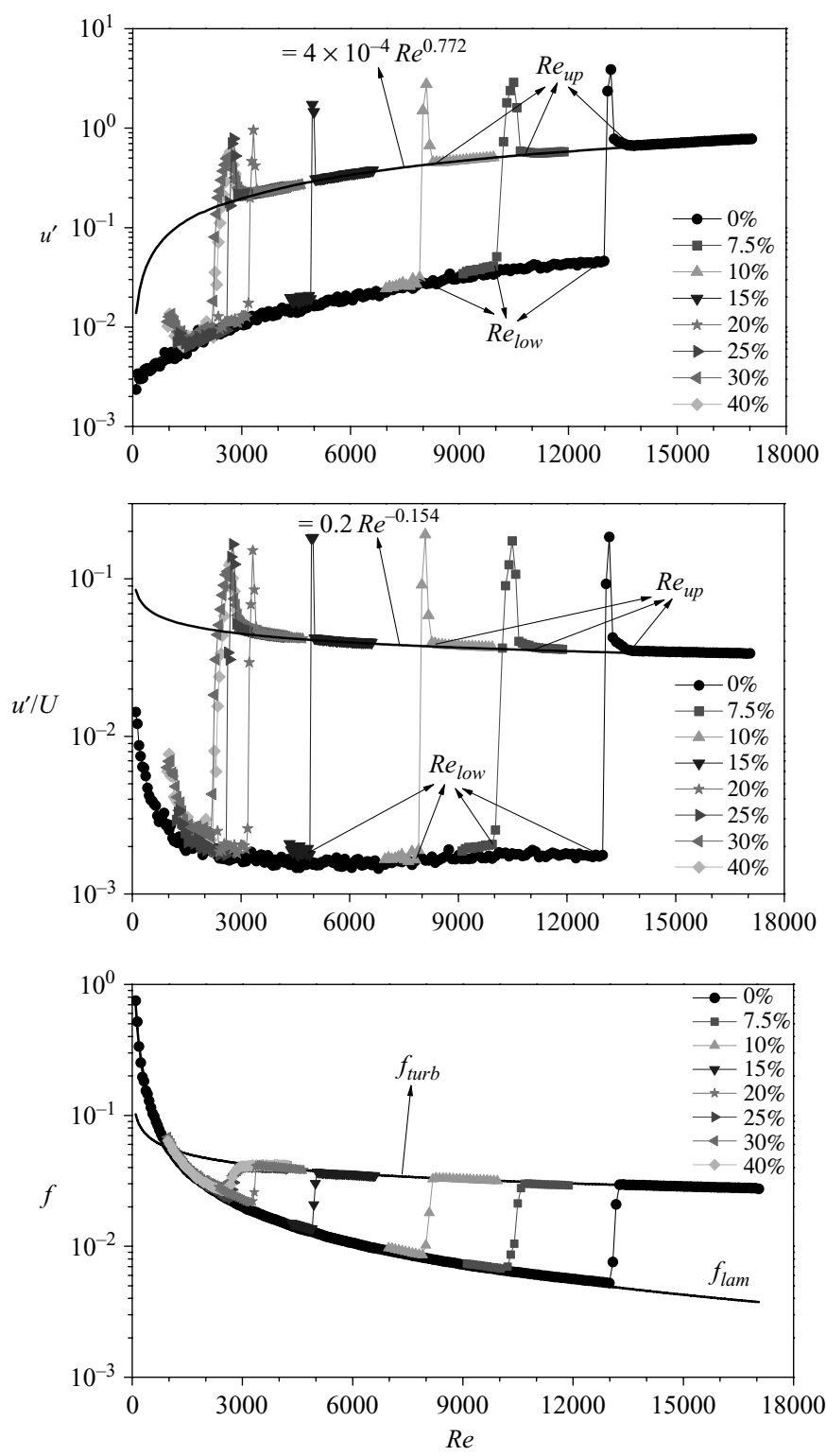

FIGURE 10. Measured r.m.s. velocity, turbulent intensity and friction factor for ring-obstacles at the pipe exit.

Reynolds number of the flow (abscissa) is clearly seen in figure 9. Furthermore the turbulent structures observed during the intermittent flow Reynolds number differ as the Reynolds number increases. For intermittent flow Reynolds number below 3000 puff-type turbulent structures were observed and for higher Reynolds number slugtype structures. This finding is also indicated in figure 9 . Figure 11 shows examples of centreline time records of puff and slug structures with velocity-time records very similar to those observed during the experiments of Wygnanski \& Champagne (1973) and Wygnanski et al. (1975). The flow characteristics of these puff and slug structures will be considered further in the next section. 


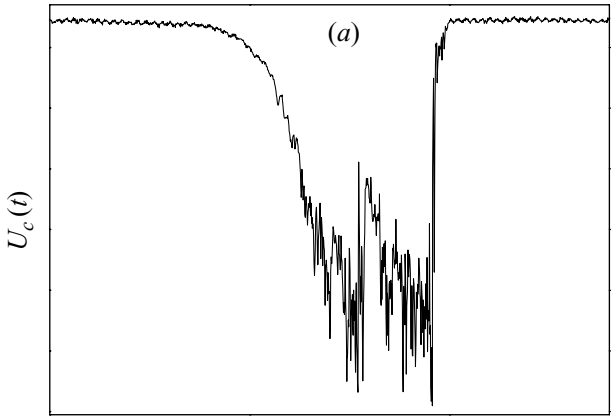

Time

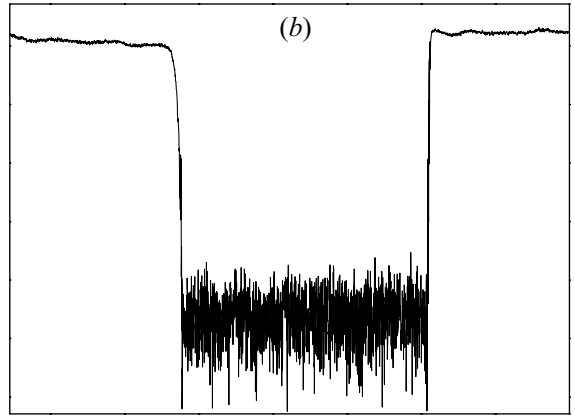

Time

Figure 11. Centreline velocity time records as examples of $(a)$ puff and $(b)$ slug-type turbulent structures.

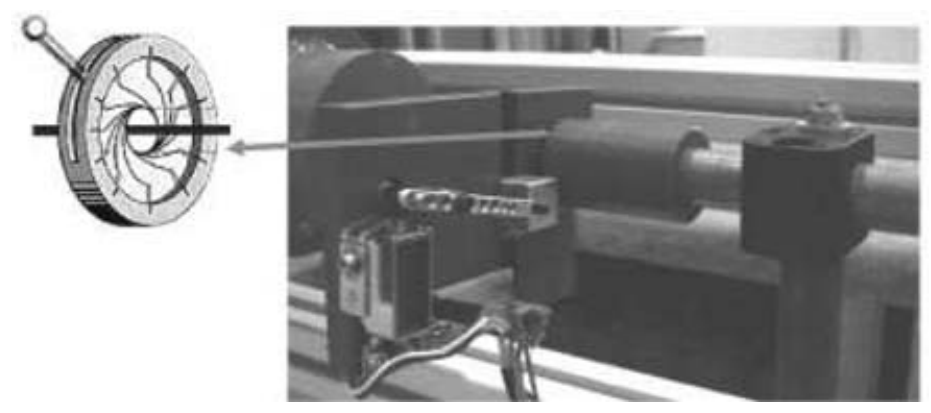

FIGURE 12. Picture of the electronically controlled iris diaphragm placed at the inlet of the pipe section.

\section{Forced transition by the iris-diaphragm}

The results presented in $\$ 2$ made it clear that naturally occurring laminar-toturbulent transition occurs in an intermittent way and in these experiments this happened at a critical Reynolds number of $R e_{c} \approx 13000$. At this Reynolds number the flow in the pipe starts to exist as a sequence of slugs that are produced in an uncontrolled way. Over a small Reynolds number range (see figure 4), the number of slugs and their individual duration are increased until the fully developed stage of turbulent pipe flow is reached.

In order to force the flow to show similar intermittent laminar-to-turbulent transition at lower Reynolds number, ring-type obstacles were employed in a sequence of experiments as described in $\S 3$. Depending on the height of the ring-type obstacles, the critical Reynolds number could be adjusted to be anywhere in the range $2000 \leqslant$ $R e_{c} \leqslant 13000$. At any adjusted Reynolds number for $R e_{c} \geqslant 3500$, the transition again took place in the form of slugs that occurred in a random manner with uncontrolled durations. Figure 10 indicates that, in principle, at a length of $L / D=666.7$, the naturally occurring and the triggered laminar-to-turbulent transitions show the same characteristic flow properties. Hence, ring-type obstacles are effective in controlling flow transition in pipe flows, i.e. to fix the critical Reynolds number for the laminarto-turbulent transition of the flow.

To provide even better control of the flow that permits the time of occurrence of turbulent slugs to be controlled, in addition to their duration, an iris diaphragm was installed at the inlet of the pipe test section as indicated in figure 12. Using 

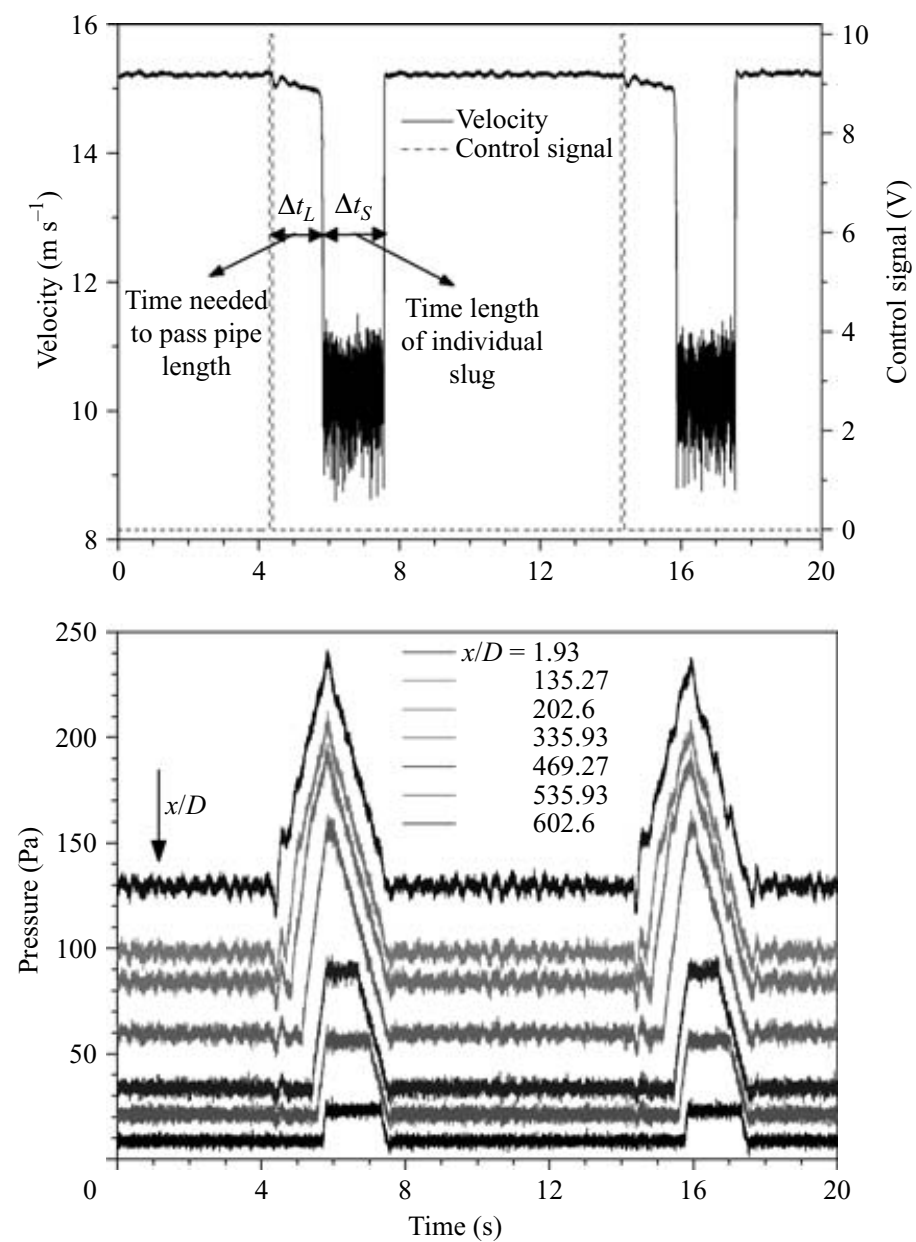

FIGURE 13. Centreline velocity and pressure records of periodically generated slug structures by the application of the iris-diaphragm triggering device.

the results in figure 9 the iris diaphragm could be used to provide conditions for the laminar-to-turbulent flow transition to occur in the form of slugs and puffs at any Reynolds number in the range $2650 \leqslant R e_{c} \leqslant 13000$. Initial experiments carried out by opening and closing the iris diaphragm confirmed this. The actual time to bring the iris diaphragm into position to represent a ring-type obstacle, i.e. to provide the required flow triggering, was adjusted to be $10 \mathrm{~ms}$. This time turned out to be short enough to produce intermittently and in a controlled manner puffs and slugs in the pipe flow, depending on the setting of the Reynolds number of the flow in accordance with the results in figure 9. Preliminary studies confirmed that the iris diaphragm triggering device produced turbulent slugs very similar to those obtained in the studies described in $\S 3$. Figure 13 shows examples of slugs produced in this way, i.e. it shows corresponding velocity records at the end of the pipe test rig and pressure-time records along the pipe, measured for $R e=5176$.

The extended test rig, sketched in figure 12, enabled sequences of puffs and slugs to be generated at different frequencies and with different durations. To trigger these flow structures, characteristic for the laminar-to-turbulent flow transition in pipes, the 
(a)
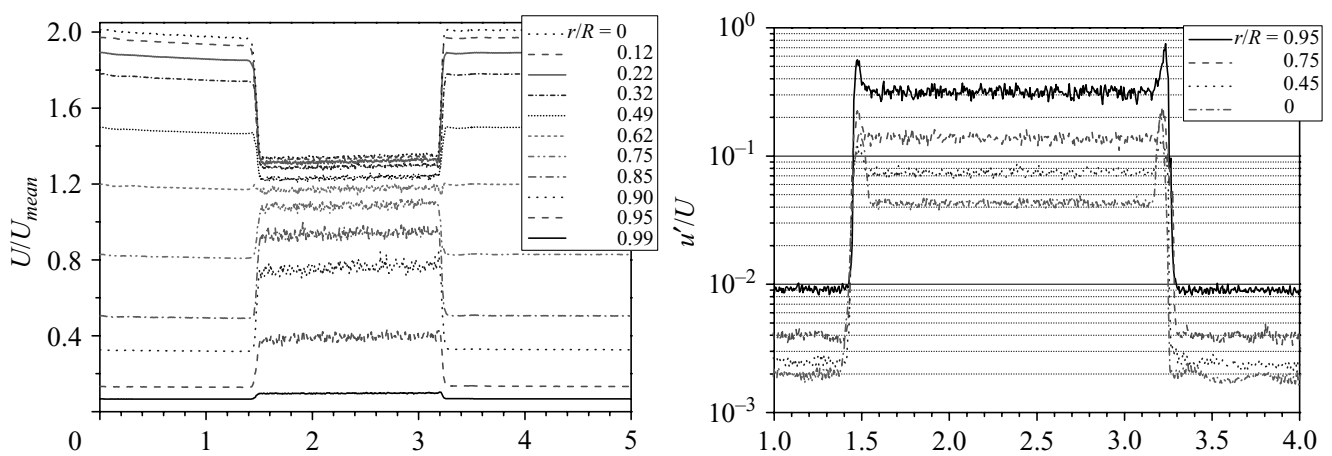

(b)
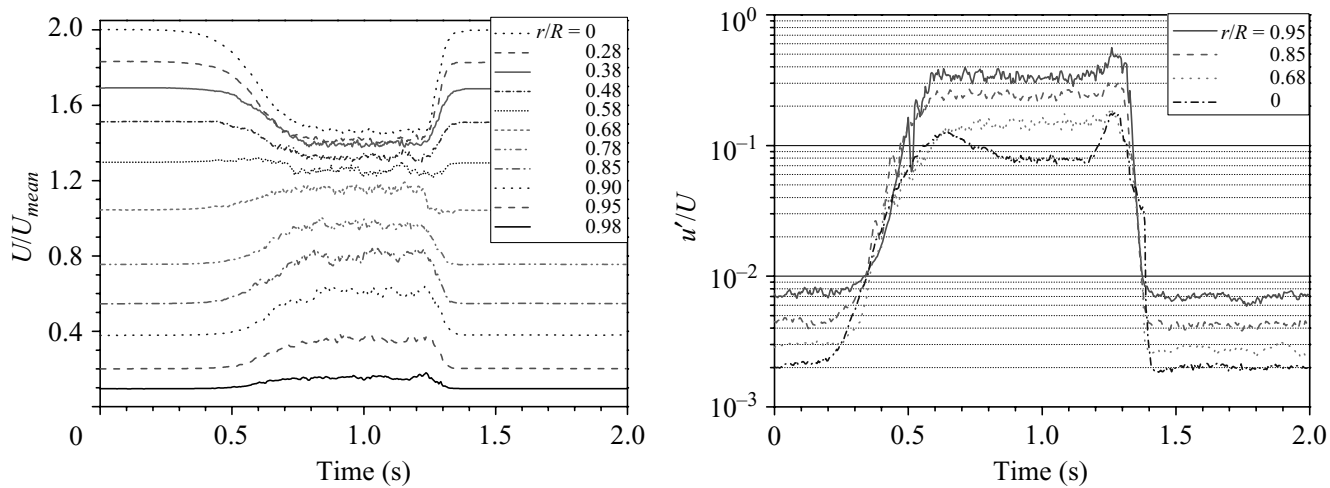

FiguRE 14. Time-averaged velocity and turbulence intensity profiles of $(a)$ a slug structure at $R e=5000$ and $(b)$ a puff structure at $R e=2850$.

results in figure 9 were used to provide the appropriate size of the iris diaphragm for each set of Reynolds number. In this way, the individual flow properties of puffs and slugs could be studied. The same time sequences of puffs and slugs could be employed to study their phase-averaged mean velocity and turbulence intensity properties. Quantities of this kind are shown in figure 14, providing the phaseaveraged mean velocity as functions of the relative time for both puffs and slugs. The corresponding turbulence intensity is also shown in figure 14. Information is provided for various radial locations indicating that both puffs and slugs occupy the whole cross-section of the pipe.

The results in figure 14 are examples chosen from a large number of time records of instantaneous velocities for puffs and slugs. The puff structure presented in figure 14(b) corresponds to $R e=2850$ and the slug structure in figure 14(a) to $R e=5000$. One of the main differences between the flow structures of puffs and slugs is in the mean velocity variations of the heads. Puffs show a decaying velocity profile in their head without the more abrupt velocity variation of the head that is typical for slugs. At the centre of the pipe, in general puffs show higher values of the local, phase-averaged turbulent intensity.

The experimental set-up also permitted the transitional cross-sectional velocity profiles to be constructed. These profiles are shown in figure 15 for the puff and slug flow structures analysed in figure 14. 

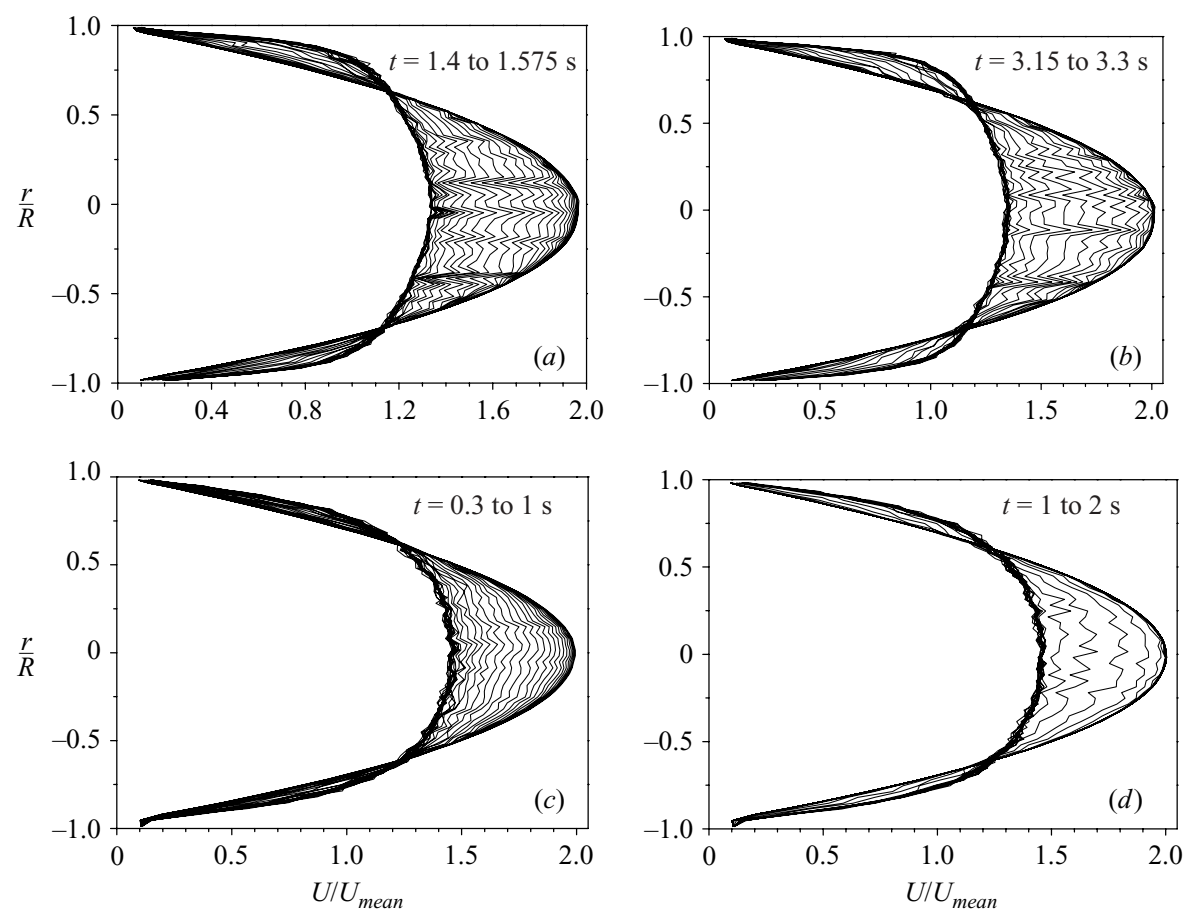

Figure 15. Cross-sectional velocity profiles with times corresponding to figure 14: $(a)$ slug at $R e=5000$, laminar-to-turbulent transition, $t=1.4-1.575 \mathrm{~s}$ of figure $14(a) ;(b)$ slug at $R e=$ 5000 , turbulent-to-laminar transition, $t=3.15-3.3 \mathrm{~s}$ of figure $14(a) ;(c)$ puff at $R e=2850$, laminar-to-turbulent transition, $t=0.3-1$ s of figure $14(b) ;(d)$ puff at $R e=2850$, turbulent-tolaminar transition, $t=1-2 \mathrm{~s}$ of figure $14(b)$.

Further information about the motion of puffs and slugs through the pipe could be deduced from the pressure records along the pipe. Especially for the slug-like flow structures, it was possible to track their motion because of the high-pressure gradients that they produced. From the pressure records, it was possible to deduce their ensemble-averaged head and tail velocities, showing that the head velocity of a slug increases during its motion through a pipe. Between the last two pressure sensors of the test section, the head and tail velocities given in figure 16 were measured and compared with results obtained by Wygnanski \& Champagne (1973) and Lindgren $(1969,1957)$. This readily suggests that the iris-diaphragm triggering device can be employed to provide puff- and slug-like flow in a well-controlled manner and so are easily accessible to detailed experimental investigations.

These investigations make it clear that the iris-diaphragm extension of the test-rig let to a flow facility that permits detailed studies of puffs and slugs because their natural appearance becomes deterministic, permitting transitional flow studies with high repeatability and reliability. Its employment is recommended for future studies of laminar-to-turbulent transition of pipe flows forced by wall-bounded obstacles.

\section{Conclusion, final remarks and outlook}

The laminar-to-turbulent transition in pipe flows occurs in form of slugs that occurred naturally in the test rig for $R e_{c} \approx 13000$. To cause slugs to occur at lower Reynolds number, ring-type obstacles were introduced into the pipe wall and at 


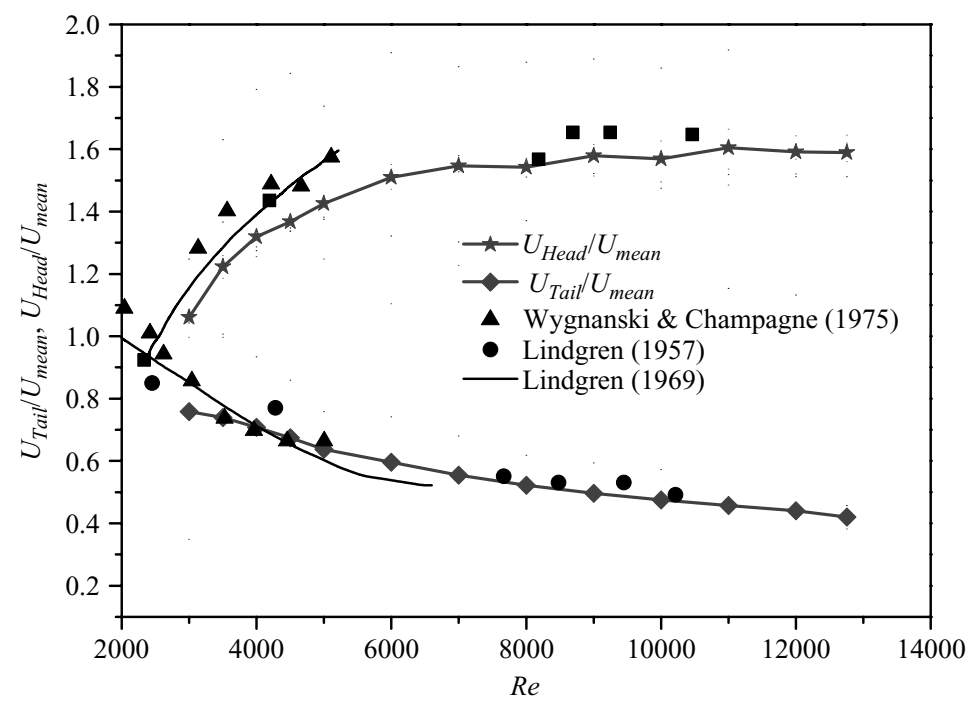

FIGURE 16. Normalized head and tail velocities of slug and puff structures with Reynolds number.

the pipe inlet. Changing the height of the obstacle permitted varying the critical Reynolds number at which the laminar-to-turbulent flow transition occurred through intermittently appearing slugs. Any Reynolds number in the range $3500 \leqslant R e \leqslant 13000$ could in this way be selected as the critical Reynolds number. The laminar-toturbulent transition through puffs could also be selected in the Reynolds number range $2000 \leqslant R e \leqslant 3500$.

To provide puff- and slug-like flow structures in a more controlled way, the test-rig was modified with an iris diaphragm. Its closing and opening were controlled by a servo-driver to produce puffs and slugs at pre-determined times for pre-selected durations. In this way, ensemble-averaged flow properties of puffs and slugs could be measured and are presented.

The iris diaphragm also permits controlled intermittency experiments where the intermittency factor can be adjusted to have any pre-chosen value between zero and one. In this way, intermittently occurring laminar and turbulent flows can be produced that are expected to show advantages when applied to heat transfer in pipe flows.

The present work received support from the DFG (Deutsche Forchunggemeinschaft), through contract number DU 101/61-1 to produce the mass flow rate control unit employed in this study. Further support was obtained through internal funding from LSTM-Erlangen. These fundings are gratefully acknowledged.

\section{REFERENCES}

Darbyshire, A. G. \& Mullin, T. 1995 Transition to turbulence in constant-mass-flux pipe flow. J. Fluid Mech. 289, 83-114.

Draad, A. A., Kuiken, G. \& Nieuwstadt, F. T. M. 1998 Laminar-turbulent transition in pipe flow for Newtonian and non-Newtonian fluids. J. Fluid Mech. 377, 267-312.

Durst, F., Heim, U., Ünsal, B. \& Kullik, G. 2003 Mass flow rate control system for time-dependent laminar and turbulent flow investigations. Meas. Sci. Technol. 14, 893-902.

Ekman, V. W. 1883 On the change from steady to turbulent motion of liquids. Ark. f. Math A 174, $1-12$. 
Hof, B., Juel, A. A. \& Mullin, T. 2003 Scaling of the turbulence transition threshold in a pipe. Phys. Rev. Lett. 91, 244502.

Lindgren, E. R. 1957 Arkiv Fys. 12, 1-8.

LindGREN, E. R. 1969 Propagation velocity of turbulent slugs and streaks in transition pipe flow. Phys. Fluids 12, 418-425.

Pfenniger, W. 1861 Transition in the inlet length of tubes at high Reynolds numbers. In Boundary Layer and Flow Control (ed. G. V. Lachman), pp. 970-980. Pergamon.

REYNOLDS, O. 1883 An experimental investigation of the circumstances which determine whether the motion of water shall be direct of sinuous, and the law of resistance in parallel channels. Phil. Trans. R. Soc. Lond. A 174, 935-982.

RoттA, J. 1956 Experimenteller Beitrag zur Entstehung Turbulenter Strömung im Rohr. Ing-Arch. 24, 258-281.

Rubin, Y., Wygnanski, I. J. \& Haritonidis, J. H. 1980 Further observations on transition in pipe. Proc. IUTAM Symp. Stuttgart, FRG, 1979, pp. 19-26. Springer.

SCHILler, L. 1934 Neu Berichte zur Turbulenzentwicklung. Z. Angew. Math. Mech. 14, 36-42.

Trefethen, L., Chapman, S., Henningson, D., Meseguer, A., Mullin, T. \& Nieuwstadt, F. 2000 Threshold amplitudes for transition to turbulence in a pipe. http://arXiv.org/abs/physics/ 0007092.

Wygnanski, I. J. \& Champagne, F. H. 1973 On transition in pipe. Part 1. The origin of puffs and slugs and the flow in a turbulent slug. J. Fluid Mech. 59, 281-351.

Wygnanski, I. J., Sokolov, M. \& Friedman, D. 1975 On transition in pipe. Part 2. The equilibrium puff. J. Fluid Mech. 69, 283-304. 